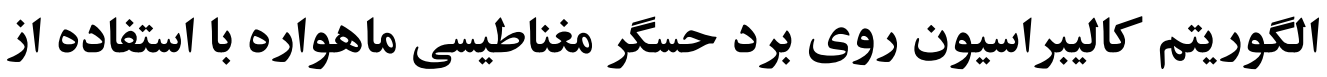

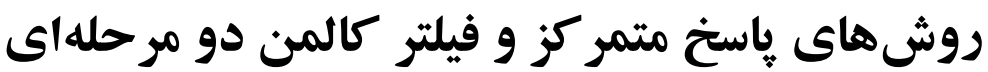

\author{
على راهدان'، حسين بلندى'، مصطفى عابدى \\ ' كارشناسى ارشد مهندسى برق، گروه كنترل، دانشكاه علم و صنعت، alirahdan1370@gmail.com

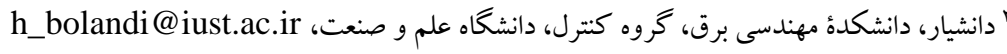

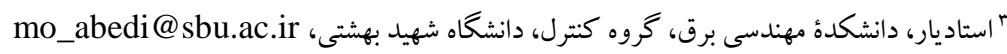

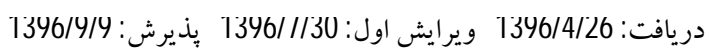

\begin{abstract}
جكيده: حسخر مغناطيسى يكى از حسخرهاى مهم مورد استفاده در سيتم تعيين و كترل وضعيت ماهو اره است. با توجه به بروز خطاهاى

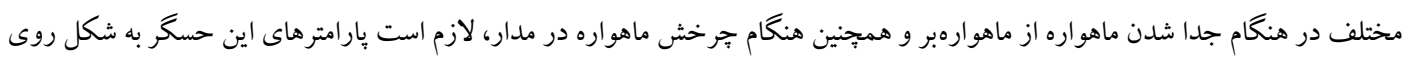

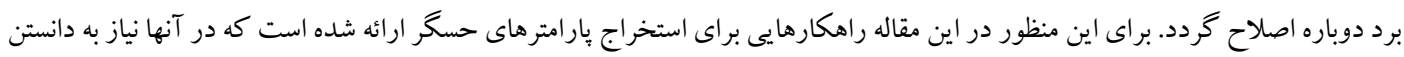

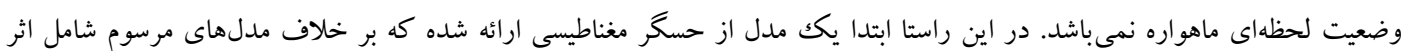
غير خطى، اثر هيستريس، اثر كو انتيزهكردن دادهها، اثر نفوذيذيرى و خطاى نصب است. در ادامه به منظور كاليبراسيون روى برد حسكر، ساختارهاى سرى دو مرحلهاى خارج از خط و برخط بيشنهاد شده است. در حالت خارج از خط، از تركيب دو الكوريتم باسخ متمركز و و لونبر كَ مار كارد استفاده شده است و در حالت برخط دو الكوريتم مبتنى بر فيلتر كالمن توسعه يافته و خنثى بيشنهاد گرديده است. با استفاده از راهكارهاى معرفى شده مى توان انواع خطاهاى حسكر شامل باياس، ضريب مقياس و خطاى نصب را بطور همزمان تعيين نمود و دقت

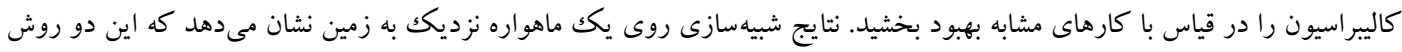

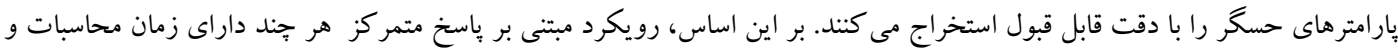

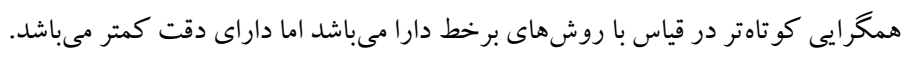
كلمات كليدى: حسكر مغناطيسى، ماهو اره، فيلتر كالمن دو مر حلهاى، الكوريتم لونبر كُ مار كاد.
\end{abstract}

\section{Design of On Board Calibration Algorithms of Satellite Magnetometer based on Two Stage Centered Solution and Kalman Filter Methods}

\author{
Ali Rahdan, Hossein Bolandi, Mostafa Abedi
}

\begin{abstract}
Magnetometer is one of the most important sensors used in the satellite attitude determination and control system. Due to occurrence of various errors when the satellite is separated from the launcher and also during its rotation in the orbit, it is necessary to re-adjust onboard the sensor parameters. For this purpose, some solutions are proposed in this paper in which the satellite current attitude is not required. In this regard, first a magnetometer model is presented that despite conventional models; it includes nonlinearity, hysteresis and data quantization effects, permeability and installation error. Then, for sensor onboard calibration purposes, two stages-offline and two-stage online series structures are suggested. In the offline case, the centered solution and Levenberg Marquardt methods have been integrated. Also, the extended and unscented Kalman filters are integrated for online case. Utilizing the suggested algorithms, different errors including bias, scale factor and installation errors are simultaneously determined and also the accuracy is improved compared to the similar works. The simulation results for a Leo satellite show that the sensor
\end{abstract}


parameters are derived with acceptable accuracy. Accordingly, it will be illustrated that the centered solution method has lower computational load and shorter time convergence, but it has lower accuracy with respect to online methodology.

Keywords: Magnetometer, satellite, two-stage Kalman filter, Levenberg Marquardt.

حسگر، مدل دقيقترى از حسگر مغناطيسى را بهدست آورده و براى

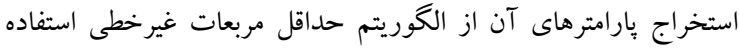

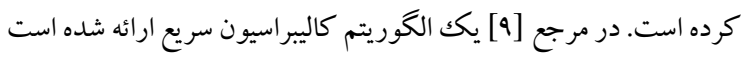
كه محاسبات كم آن، برخط بودن و همخرايى سريع باعث تمايز آن از

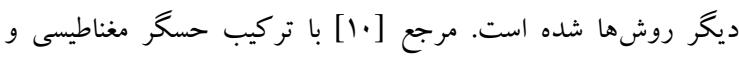

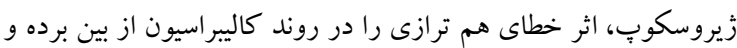
باعث افزايش دقت كاليبراسيون شده است.

يس از نصب حسكر مغناطيسى روى بدنه ماهواره و پر تاب ماهواره

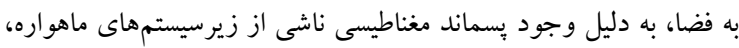

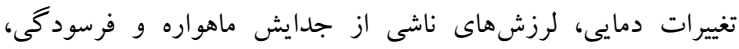

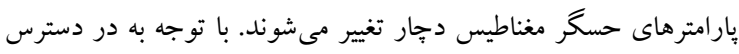

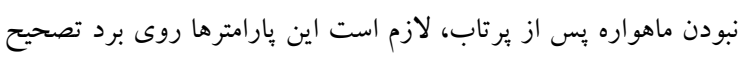

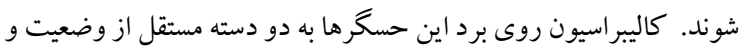

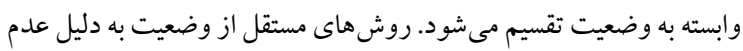
تأثيريذيرى از خطاى وضعيت داراى دقت بالاترى هستند و بيشتر مورد

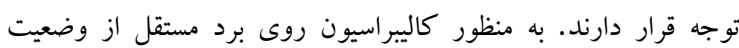

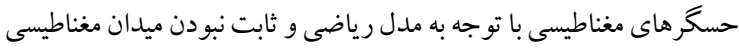

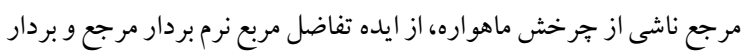

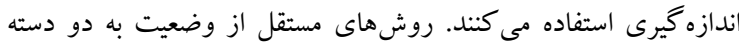

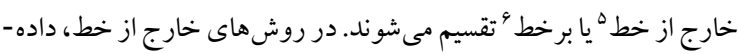

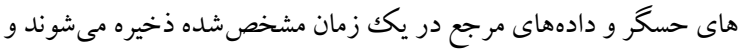

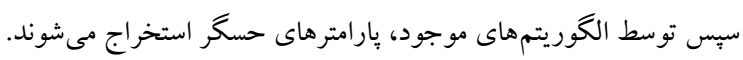

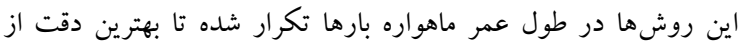
بارامترها حاصل شود. در روشهاى برخط با هر بار نمونه بردارى از

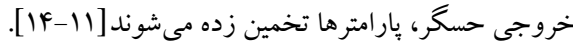
روشهاى خارج از خط مختلفى براى تعيين پيارامترهاى حسكر

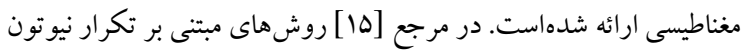

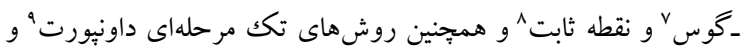

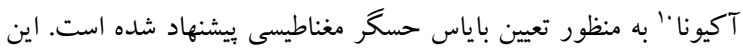

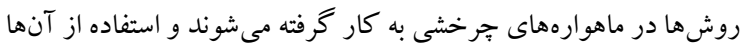

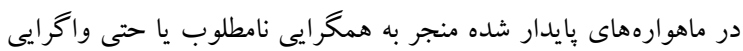

\section{1 - مقلدمه}

حسگرهاى مغناطيسى' اغلب در ماهوارههاى نزديك به زمين'

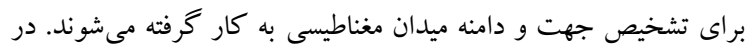

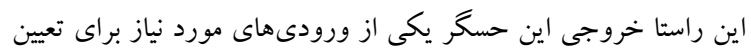

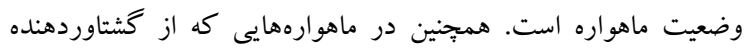

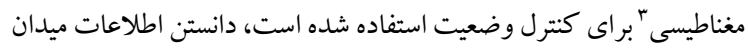

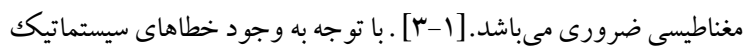

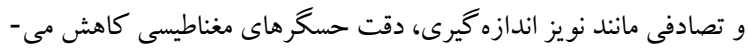

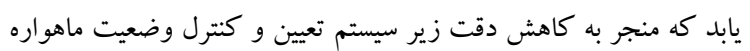

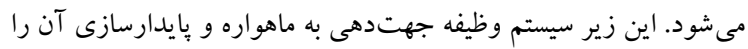

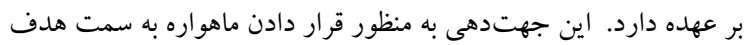

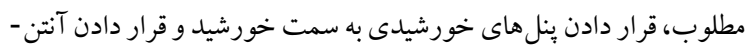

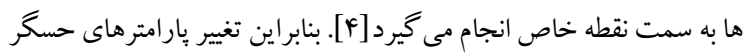

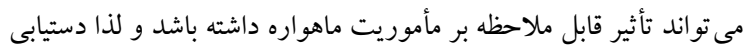

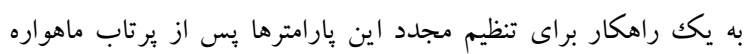

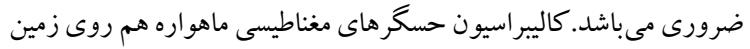

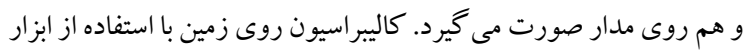

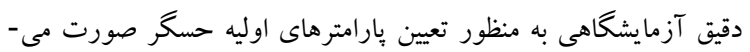

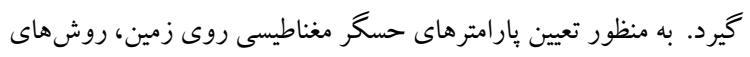

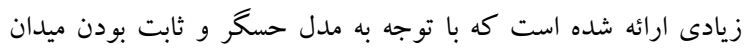
مغناطيسى مرجع در محيط آزمايشگاه، اين روشها بـ بر بايه مايه معيار تطبيق

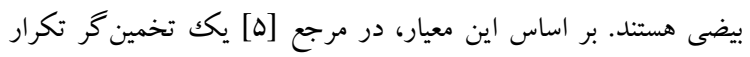

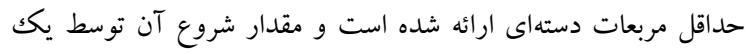

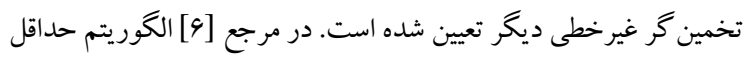

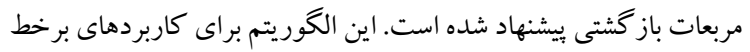

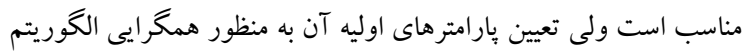

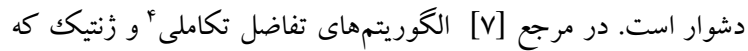

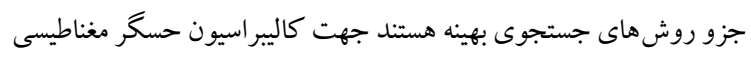

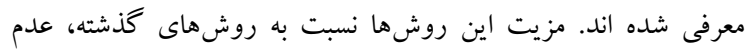

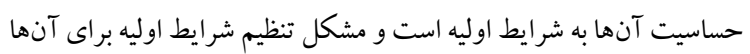

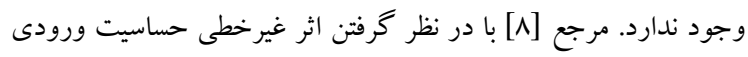

$\begin{array}{ll}{ }^{\natural} \text { Online } & { }^{\prime} \text { Magnetometer } \\ { }^{\vee} \text { Newton - Gauss } & { }^{r} \text { Low earth orbit } \\ { }^{\wedge} \text { Fix point } & { }^{r} \text { Magnetic torquer } \\ { }^{q} \text { Davenport } & { }^{\leftarrow} \text { Deferential evolution } \\ \text { 1. Acuna } & { }^{\diamond} \text { Offline }\end{array}$


محتمل در حسگر تخمين زده مىشوند. نتايج شبيهازى بهبود دقت كاليبراسيون را نسبت به كارهاى مرتبط به اثبات مىرساند. همجنين مقايسه

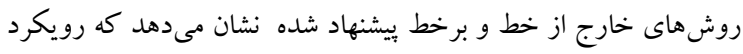

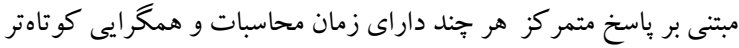

در قياس با روش هاى برخط دارا مىباشد اما داراى دقت كمتر مىباشد.

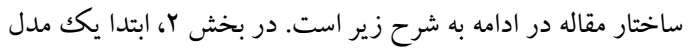

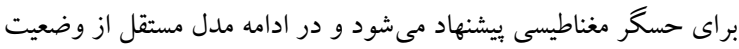

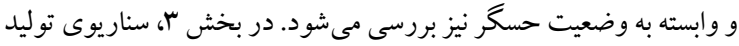

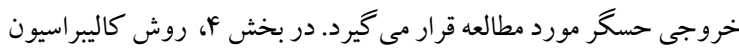

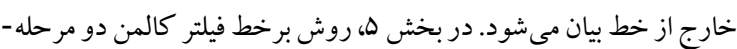

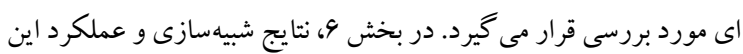

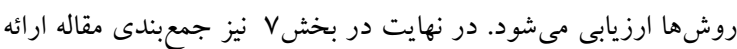
مى گردد.

\section{r- مدل بازى حسگر مغناطيسى}

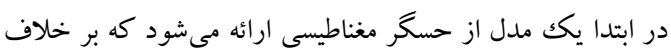

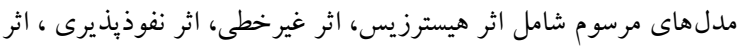

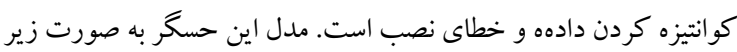

|ست[IV]

$$
\begin{aligned}
& \vec{B}_{\text {meas }}=R_{\text {body }}^{\text {sensor }}(I+L S)^{-1} \times \\
& \left(R_{\text {sensor }}^{T} \times \mathrm{C}_{\mathrm{ECI}}^{\text {body }} \times \overrightarrow{\mathrm{B}}_{E C I}+\vec{b}+\overrightarrow{\mathrm{nl}}+\overrightarrow{\mathrm{hys}}+\vec{n}+\vec{p}+\vec{q}\right)
\end{aligned}
$$

كه در آن

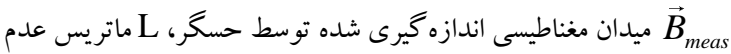
تعامد محورها، E ماتريس قطرى ضريب مقياس، حسگر، اnl اثر غير خطى بودن حسگر، نفوذيذيرى،

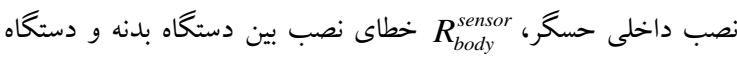

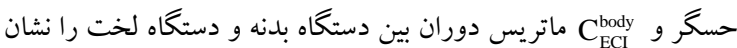

مى دهد. با توجه به ماهيت تصادفى بودن

$$
\text { وضعيت به شكل زير بهدست مى بـيد: }
$$

$\vec{B}_{\text {meas }}=R_{\text {body }}^{\text {sensor }}(I+D)^{-1} \times$

$\left(R_{\text {sensor }}^{T} \times \mathrm{C}_{\mathrm{ECI}}^{\text {body }} \times \overrightarrow{\mathrm{B}}_{\mathrm{ECI}}+\vec{b}+\vec{\varepsilon}\right)$

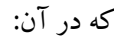

$D=L S$

$\vec{\varepsilon}=\vec{n} l+\overrightarrow{h y s}+\vec{q}+\vec{n}$
مى شود. در مرجع [19] روش باسخ متمر كز' براى بهدست آوردن باياس،

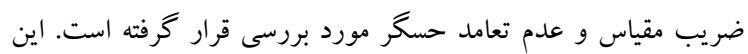

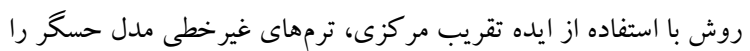

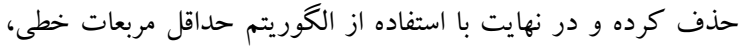

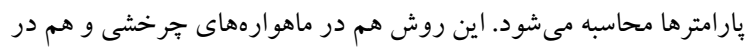

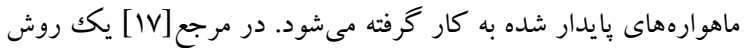
دو مرحلهاى ك كه تكميل كننده روش ياسخ متمر كز است ارائه شده است.

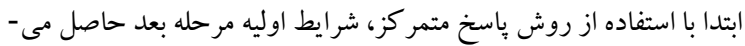

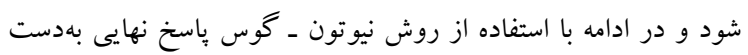
مى آيد.

روش هاى بر خط اغلب بر بايه فيلتر كالمن هستند. در مرجع[11]

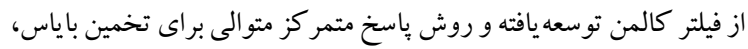

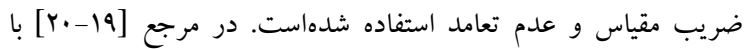

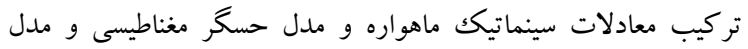

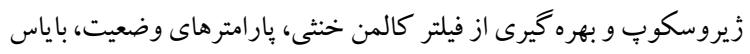

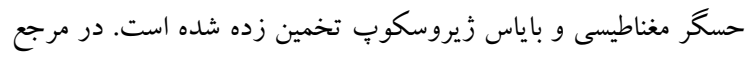

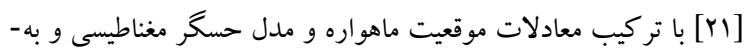

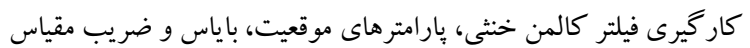
حسگر مغناطيسى بهدست آورده شده است. در [rY] با ارائه الكوريتم

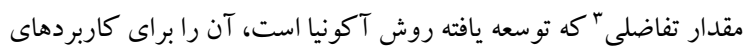

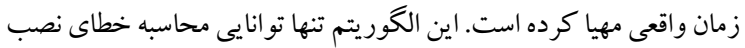
رادارد. در اين مقاله نيز با توجه به اهميت تعيين بارامترهاى حسخر بس از

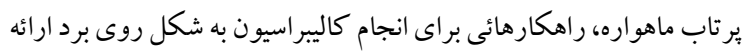

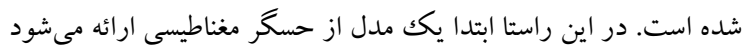

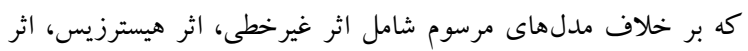

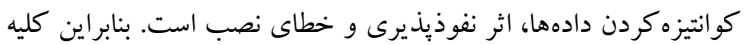

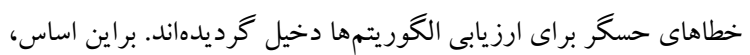
يكك روش خارج از خط جديد از تركيب دو الكَوريتم باسخ متمركز و و

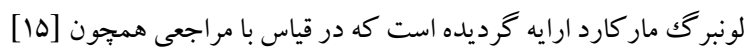
و [19] امكان محاسبه همزمان باياس، ضريب مقياس،عدم تعامد را فراهم

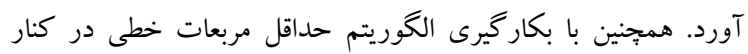

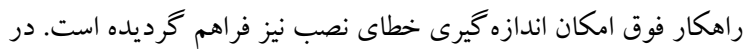

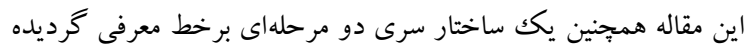

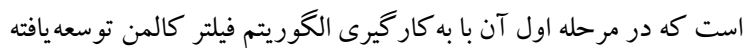
يا خنثى، باياس، ضريب مقياس و عدم تعامد حسكر محاسبه مى شود و در

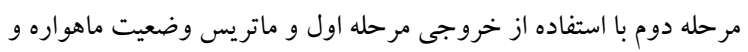

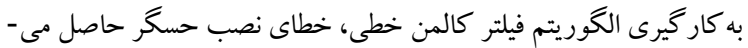

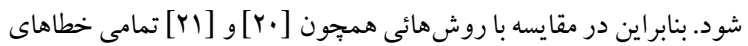

${ }^{r}$ Differential value
' Centered solution

$r$ Two step 
كه در آن U ماتريس متعامد و V ماتريس قطرى با المان هاى V v و

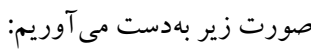
$w_{i}=-1+\sqrt{1+v_{i}} \quad i=1,2,3$

(IV)

در نهايت

$D=U \operatorname{diag}\left(\mathrm{w}_{\mathrm{i}}\right) U^{T}$

$\vec{b}=(I+D)^{-1} \vec{c}$

\section{r- سيستم هاى توصيف كننده حر كت ماهواره}

مطابق با شكل (1) سيستمهاى مختصات استفاده شده براى توصيف

حركت ماهواره عبارت است از [rr]:

سيستم مختصات بدنه': مبدا آن مركز جرم ماهواره است

ومحورهاى آن منطبق بر محورهاى اصلى است.

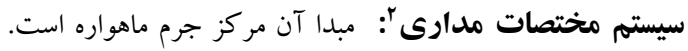

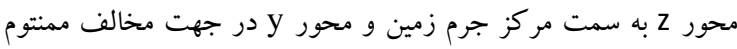

زاويهاى صفحه مدار و محورX توسط قانون دست راست تعيين مى شود.

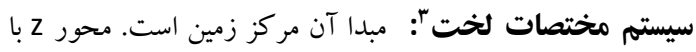

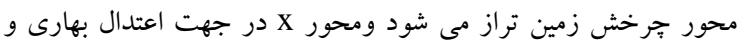

محورل توسط قانون دست راست تعيين مى شود.

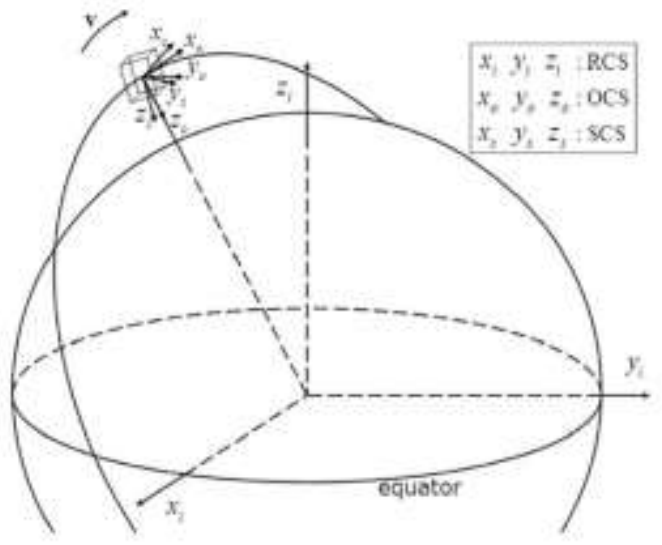

شكل ا: سيستمهاى مختصات توصيف حر كت ماهواره [YY]

\section{ع- سناريوى توليد خروجى حسكر مغناطيسى}

مطابق با شكل (Y) براى توليد خروجى حسكر مغناطيسى بايد ماتريس

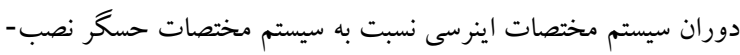

شده روى بدنه ماهواره و همجنين اطلاعات ميدان مغناطيسى مرجع را

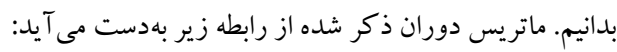

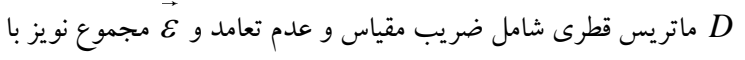
ميانگين صفر و كواريانس \ است. با كم كردن اندازه دو ميدان از يكديخر مدل مستقل از وضعيت حسخر به صورت زير بهدست مئ بـ آيد: $y=\left\|\vec{B}_{\text {meas }}\right\|^{2}-\left\|\vec{B}_{E C I}\right\|^{2}=-\vec{B}_{\text {meas }}{ }^{T}\left(2 D+\|D\|^{2}\right) \vec{B}_{\text {meas }}$

$+2 \overrightarrow{\mathrm{B}}_{\text {meas }}{ }^{\mathrm{T}}(I+D) \vec{b}-\|\vec{b}\|^{2}+v$

كه در آن: - م

$v=2\left((I+D) \vec{B}_{\text {meas }}-\vec{b}\right)^{T} \vec{\varepsilon}-\|\vec{\varepsilon}\|^{2}$

نويز اين مدل داراى ميانخين ( $)$ ) و واريانس( $\mu=-\operatorname{tr}(\Sigma)$

$\sigma^{2}=4\left((I+D) \vec{B}_{\text {meas }}-\vec{b}\right)^{T} \Sigma\left((I+D) \vec{B}_{\text {meas }}-\vec{b}\right)$

$+2\left(\operatorname{tr}\left(\Sigma^{\mathrm{T}} \Sigma\right)\right)$

براى كاهش ميزان غيرخطى بودن مدل، متغيرهاى زير تعريف مى گردند: $E=2 D+D^{\mathrm{T}} D=\left(\begin{array}{lll}E_{11} & E_{12} & E_{13} \\ E_{12} & E_{22} & E_{23} \\ E_{13} & E_{23} & E_{33}\end{array}\right)$

$\vec{c}=(I+D) \vec{b}$

با در نظر گرفتن عناصر ماتريس E به صورت بردارى، خواهيم داشت:

$\vec{F}=\left[\begin{array}{llllll}E_{11} & E_{22} & E_{33} & E_{12} & E_{13} & E_{23}\end{array}\right]^{T}$

بر اين اساس، مدل اصلاح شده به صورت زير بهدست آورده مىشود: $y=\vec{L}^{\mathrm{T}} x^{\prime}-\left\|\vec{b}\left(x^{\prime}\right)\right\|^{2}+v$

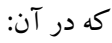

$x^{\prime}=\left[\begin{array}{ll}c^{T} & \vec{F}^{T}\end{array}\right] T$

$\vec{L}=\left[\begin{array}{ll}2 \vec{B}_{\text {meas }}^{\mathrm{T}} & -\overrightarrow{\mathrm{B}}^{T}\end{array}\right]^{\mathrm{T}}$

$\vec{B}=\left[B_{\text {meas } 1}^{2} B_{\text {meas } 2}^{2} B_{\text {meas } 3}^{2} 2 B_{\text {meas } 1} B_{\text {meas } 2}\right.$

$\left.2 B_{\text {meas } 1} B_{\text {meas } 3} 2 B_{\text {meas } 2} B_{\text {meas } 3}\right]^{T}$

انديسهاى ا، rو rا، شماره عنصر بردار

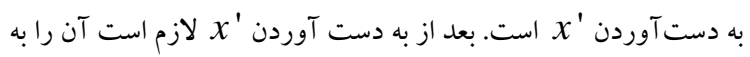
آن و D ت تبديل كنيم. به دليل متقارن بودن ماتريس E، تجزيه مقدار تكين آن به صورت زير است:

$E=U V U^{T}$ 
0- روش خارج از خط حداقل مربعات دو

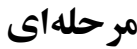

مطابق با شكل (r) براى بهدست آوردن پيارامترهاى حسكر با دقت

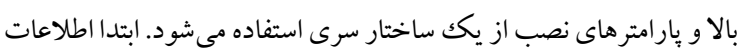
ميدان مغناطيسى مرجع

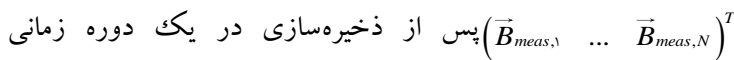

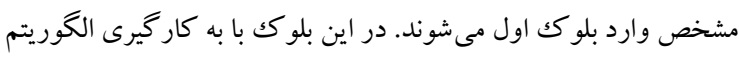

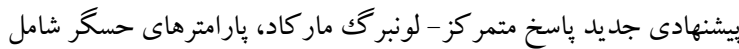

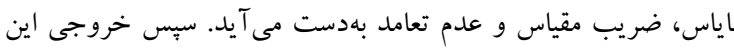

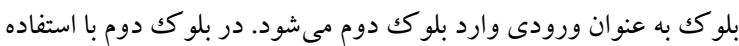
از خروجى بلوك قبل و و اطلاعات ماتريس وضعيت

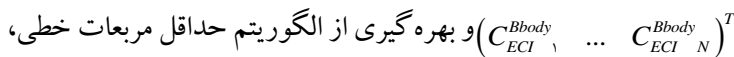

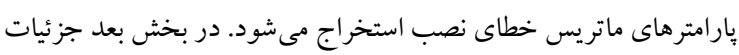
هر يك از اين بلو ككها بيان شده است. بان.

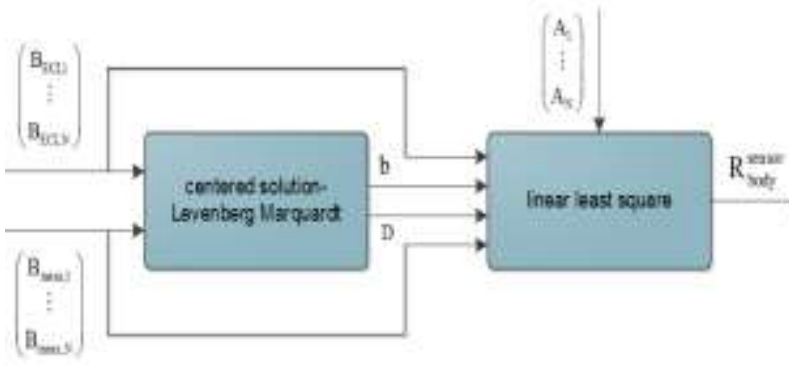

شكل r: روش حداقل مربعات دو مر حلهاى

ه-1 - مرحله اول از روش حداقل مربعات دو مرحلهاى در اين مر حله ابتدا با تعريف متغيرهاى مر كزى و متغيرهاى متمركز،

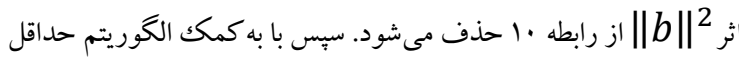

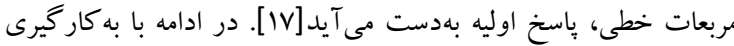

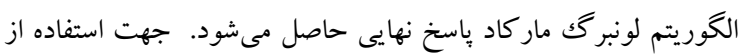

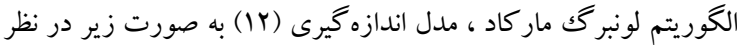

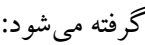
$y_{k}=\vec{L}_{k}^{\mathrm{T}} x^{\prime}-\left\|\vec{b}\left(x^{\prime}\right)\right\|^{2}+v_{k}=h_{k}\left(x^{\prime}\right)+v_{k}$

با در نظر مقدار X به عنوان شرايط اوليه الكوريتم، ماتريس زاكوبى از

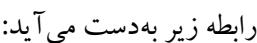
$\mathbf{H}_{k}=\frac{\partial h_{k}\left(x^{\prime}\right)}{\partial x^{\prime}}\left(x_{k}\right)=\vec{L}_{k}^{\mathrm{T}}-\frac{\partial\left\|\vec{b}\left(x^{\prime}\right)\right\|^{2}}{\partial x^{\prime}}\left(x_{k}\right)$

$$
C_{\text {inertial }}^{\text {body }}=C_{\text {inertial }}^{\text {orbit }} \times C_{\text {orbit }}^{\text {body }}
$$

ماتريس دوران بين سيستم مختصات اينرسى و سيستم $C_{\text {inertial }}^{\text {orbit }}$ مختصات مدارى است كه تابعى از موقعيت و سرعت خطى ماهواره است و از رابطه زير بهدست مى آيد [rr]:

$$
C_{\text {inertial }}^{\text {orbit }}=\left[\begin{array}{lll}
o_{1} & O_{2} & o_{3}
\end{array}\right]
$$

كه در آن: - م

$$
\begin{aligned}
& o_{3}=\frac{r}{\|r\|} \\
& o_{2}=\frac{r \times v}{\|r \times v\|} \\
& o_{1}=o_{3} \times o_{2}
\end{aligned}
$$

rr بردار موقعيت ماهواره و v بردار سرعت خطى ماهو اره است. براى توليد اين دو بردار نياز به مدلسازى ديناميك مدارى ماهواره است.

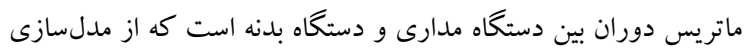
ديناميك و سينماتيك وضعيت ماهو اره بهدست ميى دآيد. جهت تعيين ميدان مغناطيسى مرجع جندين مدل ارائه شده است

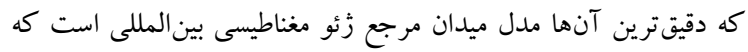

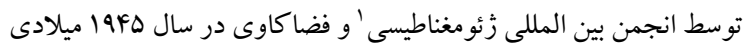

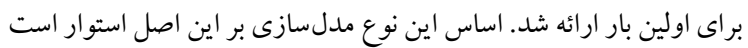

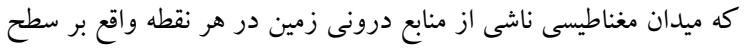

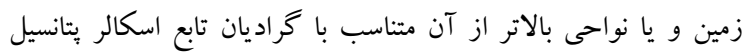

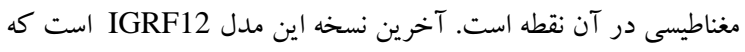

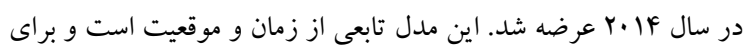

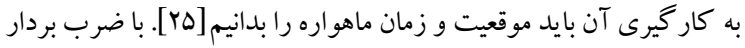

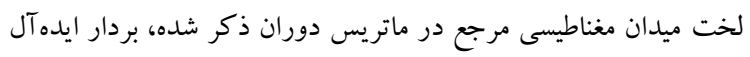

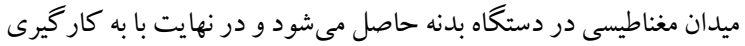
مدل حسخر، خروجى آن بهدست مى آيد.

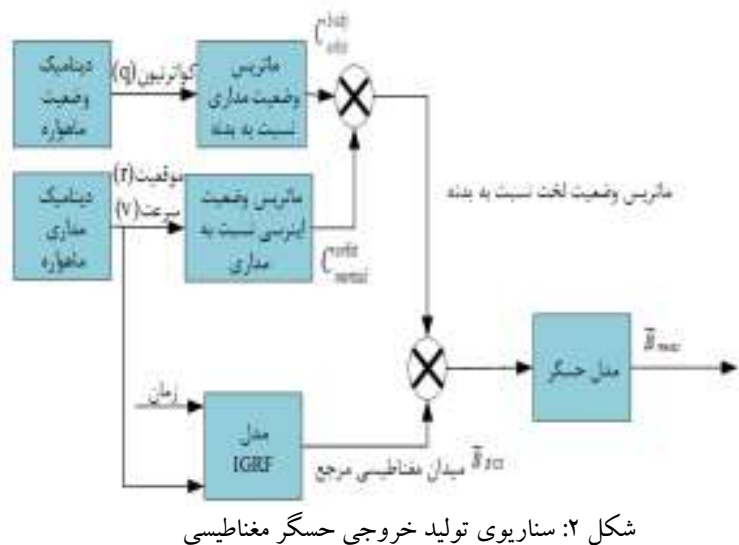




$$
\vec{B}_{\text {meas }}=R_{\text {body }}^{\text {sensor }} \vec{B}_{\text {body }}+\vec{\eta}
$$

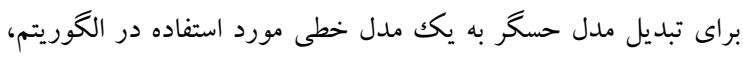

$$
\text { متغيرهاى زير تعريف مىشوند: }
$$

$$
\begin{aligned}
& W=\left(\begin{array}{ccccccccc}
B_{b o d y 1} & B_{b o d y 2} & B_{b o d d y 3} & 0 & 0 & 0 & 0 & 0 & 0 \\
0 & 0 & 0 & B_{b o d y 1} & B_{b o d y 2} & B_{b o d y 3} & 0 & 0 & 0 \\
0 & 0 & 0 & 0 & 0 & 0 & B_{b o d y 1} & B_{b o d y 2} & B_{b o d d y 3}
\end{array}\right) \\
& \vec{V}=\vec{B}_{\text {meas }} \\
& x=\left(\begin{array}{lll}
r_{11} & \ldots & r_{33}
\end{array}\right)^{T}
\end{aligned}
$$$$
\text { انديس هاى 1، Y و rا، شماره عنصر بردار }
$$

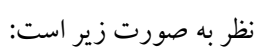$$
\vec{V}=W x+\vec{\eta}
$$

با توجه به وجود N داده اندازهيرى، ماتريس Wو

$$
\text { صورت زير تعريف مى گردند: }
$$

$$
W=\left(\begin{array}{c}
W_{1} \\
\vdots \\
W_{N}
\end{array}\right)_{3 N \times 3}
$$

$$
V=\left(\begin{array}{c}
\vec{V}_{1} \\
\vdots \\
\vec{V}_{N}
\end{array}\right)_{3 N \times 1}
$$

$$
R^{\prime}=\left(\begin{array}{ccc}
(I+D)^{-1} \Sigma_{1} & 0 & 0 \\
0 & \ddots & 0 \\
0 & 0 & (I+D)^{-1} \Sigma_{N}
\end{array}\right)
$$

در نهايت ياسخ حداقل مربعات خطى براى محاسبه $x$ به صورت زير

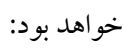
$x=\left(W^{T} R^{-1} W+\gamma I\right)^{-1} W^{T} R^{-1} V$

$$
\text { كه ريك ضريب ثابت مثبت است. }
$$

\section{7- روش برخط فيلتر كالمن دو مرحلهاى}

مطابق با شكل (F) اطلاعات ميدان مغناطيسى مرجع خروجى حسكر اول مىشوند. در اين بلوكك با به كار گيرى الكوريتم فيلتر كالمن توسعه -

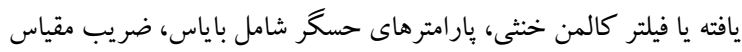

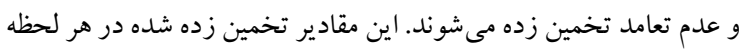
به عنوان ورودى وارد مرحله دوم مى شوند. در لايه دوم با استفاده از

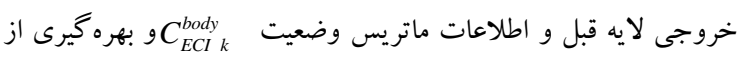

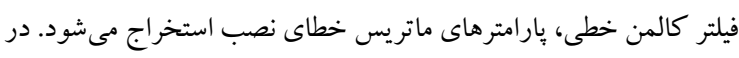

$\frac{\partial\left\|\vec{b}\left(x^{\prime}\right)\right\|^{2}}{\partial c_{m}}=\left(2(I+E)^{-1} \vec{c}\right)_{m}$

$\frac{\partial\left\|\vec{b}\left(x^{\prime}\right)\right\|^{2}}{\partial E_{m n}}=-\left(2-\delta_{m n}\right)\left((I+E)^{-1} \vec{c}\right)_{m} \times$

(rq) $\quad\left((I+E)^{-1} \vec{c}\right)_{n}$

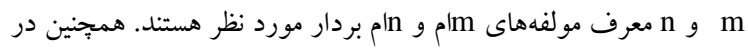

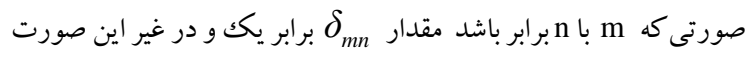

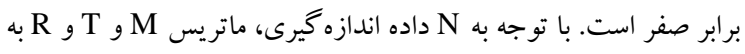
صورت تعريف مى گردند: $M=\left(\begin{array}{c}H_{1} \\ \vdots \\ H_{N}\end{array}\right)$

$\boldsymbol{T}=\left(\begin{array}{c}\boldsymbol{y}_{1}-\boldsymbol{h}_{1}\left(\boldsymbol{x}_{\mathrm{k}}\right) \\ \vdots \\ \boldsymbol{y}_{N}-\boldsymbol{h}_{N}\left(\boldsymbol{x}_{\mathrm{k}}\right)\end{array}\right)$

$R=\left(\begin{array}{ccc}\sigma_{1}^{2} & 0 & 0 \\ 0 & \ddots & 0 \\ 0 & 0 & \sigma_{N}^{2}\end{array}\right)$

بر اين اساس ميزان تغييرات بارامترها از معادله زير حاصل مىشود: $\Delta x=\left(M^{T} R^{-1} M\right)^{-1} M^{T} R^{-1} \mathrm{~T}$ در نهايت يارامترها با استفاده از رابطه زير به روز مىشوند: $x_{\mathrm{k}+1}=x_{\mathrm{k}}+\Delta x$ روابط (Y9) تا (r) آنقدر تكرار مىشوند تا الكوريتم همخر اشود. شرط

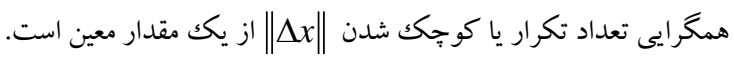
ه-r- مرحله دوم از الكوريتم حداقل مربعات اين مر حله براى استخر اج يارامترهاى خطاى نصـب مورد استفاده قرار

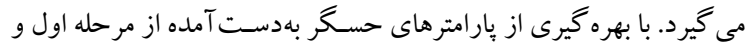

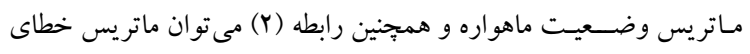

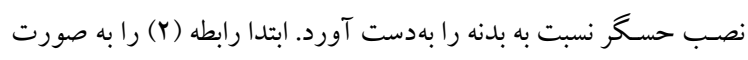

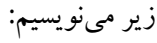
$\vec{B}_{\text {meas }}=R_{\text {body }}^{\text {sensor }}(I+D)^{-1}\left(C_{E C I}^{\text {Bbody }} \times \vec{B}_{E C I}+\vec{b}\right)$ $+R_{\text {body }}^{\text {sensor }}(I+D)^{-1} \vec{\varepsilon}$

$$
\begin{aligned}
& \text { در ادامه متغيرهاى زير تعريف مىشوند: } \\
& \vec{B}_{b o d y}=(I+D)^{-1}\left(C_{E C I}^{B b o d y} \times \vec{B}_{E C I}+\vec{b}\right) \\
& R_{b o d y}^{\text {sensor }}(I+D)^{-1} \vec{\varepsilon} \approx(I+D)^{-1} \vec{\varepsilon}=\vec{\eta} \\
& R_{b o d y}^{\text {sensor }}=\left(\begin{array}{lll}
r_{11} & r_{12} & r_{13} \\
r_{21} & r_{22} & r_{23} \\
r_{31} & r_{32} & r_{33}
\end{array}\right)
\end{aligned}
$$


$(\Delta F)$

$$
\begin{aligned}
& J=\left[\begin{array}{llll}
B_{\text {meas } 1} b_{1} & B_{\text {meas } 2} b_{2} & B_{\text {meas } 3} b_{3} \\
B_{\text {meas } 1} b_{2}+B_{\text {meas } 2} b_{1} & B_{\text {meas }} b_{3}+B_{\text {meas } 3} b_{1} & B_{\text {meas } 2} b_{3}+B_{\text {meas } 3} b_{2}
\end{array}\right]
\end{aligned}
$$

روابط (اله) تا (هاه) آنقدر تكرار مىشوند تا الخوريتم به مقدار مطلوب

$$
\text { همخرا شود. }
$$

$$
\text { 9-1-r- الكوريتم فيلتر كالمن خنثى }
$$

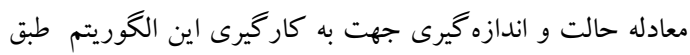

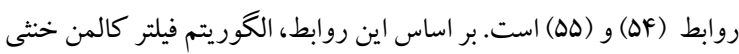

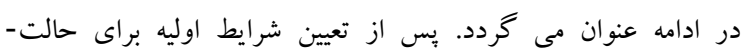

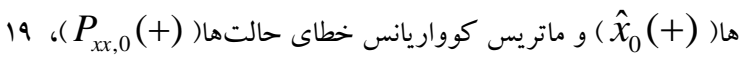

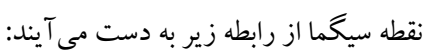

$$
\chi_{i, k}=\left\{\begin{array}{cc}
\hat{x}_{k}(+) & i=0 \\
\hat{x}_{k}(+)+\left(\sqrt{(9+\lambda) P_{x x, k}(+)}\right)_{i} & i=1, \ldots, 9 \\
\hat{x}_{k}(+)-\left(\sqrt{(9+\lambda) P_{x x, k}(+)}\right)_{i} & i=10, \ldots, 18
\end{array}\right.
$$

كه (+)

$$
\begin{aligned}
& w_{i}^{(m)}= \begin{cases}\frac{\lambda}{9+\lambda} & i=0 \\
\frac{1}{2(9+\lambda)} & i=1, \ldots, 18\end{cases} \\
& w_{i}^{(c)}= \begin{cases}\frac{\lambda}{9+\lambda}+\left(1-\alpha^{2}+\beta\right) \quad i=0 \\
\frac{1}{2(9+\lambda)} & i=1, \ldots, 18\end{cases}
\end{aligned}
$$

$$
\lambda=\alpha^{2}(9+\kappa)-n
$$

در اين روابط $\lambda$ بارامتر مقياس، $\alpha$ توزيع نقاط سيخما، $\beta$ دانش قبلى

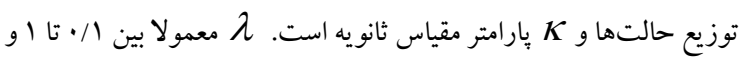

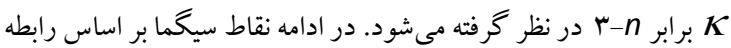

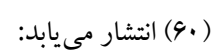

$$
\chi_{i, k+1}=\chi_{i, k}
$$

حالتهاى ييشين و ماتريس كواريانس خطاى حالت بيشين به صورت زير

$$
\begin{aligned}
& x_{k+1}(-)=\sum_{i=0}^{18} w_{i}^{(m)} \chi_{i, k+1} \\
& P_{x x, k+1}(-)=\sum_{i=0}^{18} w_{i}^{(c)}\left(\chi_{i, k+1}-\hat{x}_{k+1}(-)\right)\left(\chi_{i, k+1}-\hat{x}_{k+1}(-)\right)^{T}
\end{aligned}
$$

$$
\text { يس از آن معادله اندازه گيرى به شكل زير انتشار مى يابد: }
$$

$z_{i, k+1}=h\left(\chi_{i, k+1}\right)$

$\left(\boldsymbol{q u}^{4}\right)$
بخش بعد جزييات هر يك از اين مراحل بيان شده است.

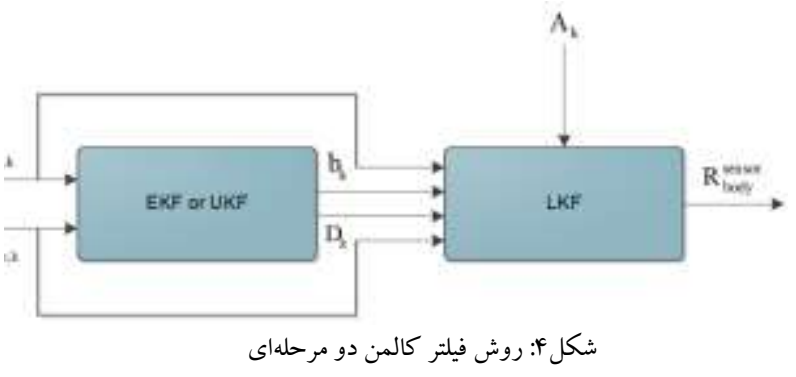

9-1- مرحله اول از الكوريتم فيلتر كالمن دو مرحلهاى 9-1-1- الكوريتم فيلتر كالمن توسعه يافته جهت به كار گيرى فيلتر كالمن توسعه يافته بايد معادله حالت و معادله

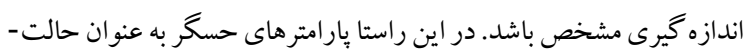

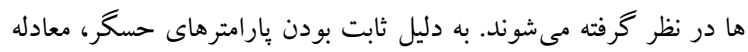

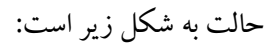

$$
x(k+1)=x(k)
$$

با توجه به رابطه (Y)، معادله اندازهيرى به صورت رابطه (F) درنظر گَرفته مى شود: - مى $y=\left\|\vec{B}_{\text {means }}\right\|^{2}-\left\|\vec{B}_{E C I}\right\|^{2}=-\vec{B}_{\text {means }}{ }^{T}\left(2 D+D^{2}\right) \vec{B}_{\text {means }}$ $+2 \vec{B}_{\text {means }}{ }^{T}(I+D) \vec{b}-\|b\|^{2}+v=h(x)+v$

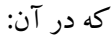

$$
x=\left[\begin{array}{lllll}
\vec{b}^{T} & D_{11} D_{22} D_{33} D_{12} D_{13} D_{23}
\end{array}\right]^{T}
$$

بر اساس روابط (F)) و (F9) ، الكوريتم فيلتر كالمن توسعه يافته به صورت زير طراحى مى نود:

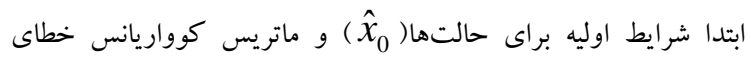

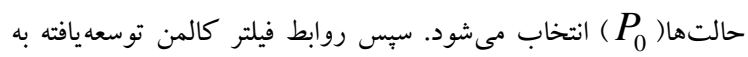
$K_{k}=P_{k} H_{k+1}{ }^{T}\left(\hat{x}_{k}\right)\left(H_{k+1}\left(\hat{x}_{k}\right) P_{k} H_{k+1}{ }^{T}\left(\hat{x}_{k}\right)\right.$ $\left.+\sigma_{k+1}^{2}\left(\hat{x}_{k}\right)\right)^{-1}$

$$
\hat{x}_{k+1}=\hat{x}_{k}+K_{k}\left(y_{k+1}-h_{k+1}\left(\hat{x}_{k}\right)\right)
$$

$P_{k+1}=\left(I-K_{k} H_{k+1}\left(\hat{x}_{k}\right)\right) P_{k}$

در روابط بالا ماتريس زاكوبى به صورت زير بهدست مى آيد: $H(x)=\frac{\partial h(x)}{\partial x}=$

$\left[2 B_{\text {meas }}{ }^{T}(I+D)-2 b^{T}-S^{T} M_{E D}(D)+2 J\right]$

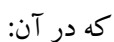
$M_{E D}(D)=2 I+\left(\begin{array}{cccccc}2 D_{11} & 0 & 0 & 2 D_{12} & 2 D_{13} & 0 \\ 0 & 2 D_{22} & 0 & 2 D_{12} & 0 & 2 D_{23} \\ 0 & 0 & 2 D_{33} & 0 & 2 D_{13} & 2 D_{23} \\ D_{12} & D_{12} & 0 & D_{11}+D_{22} & D_{23} & D_{13} \\ D_{13} & 0 & D_{13} & D_{23} & D_{11}+D_{33} & D_{12} \\ 0 & D_{23} & D_{23} & D_{13} & D_{12} & D_{22}+D_{33}\end{array}\right)$ 
استفاده مىشود. مشخصات اين حسخر در جدول (1) آورده شده است. در

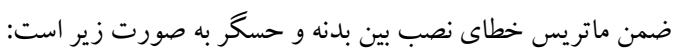
$R=\left(\begin{array}{ccc}0.9992 & 0.0349 & -0.0175 \\ -0.0346 & 0.9992 & 0.0174 \\ 0.0180 & -0.0168 & 0.9997\end{array}\right)$

$(V)$

شكل (ه) مجموع باياس و نويز حسكر مغناطيسى را نشان مىدهد و شكل

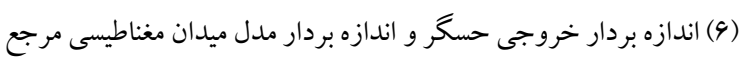

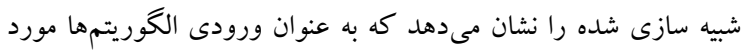

استفاده قرار مى گيرد.

جدول ا: مشخصات حسخر مغناطيسى AMR-RS422-LV

\begin{tabular}{|c|c|c|c|}
\hline \multicolumn{3}{|c|}{ مقدار } & يار امتر \\
\hline \multicolumn{3}{|c|}{$(1 \cdots, \wedge \cdots, q \cdots) \mathrm{nT}$} & مجموع باياس(باياس+رانش باياس) \\
\hline \multicolumn{3}{|c|}{$\Gamma_{n T / \operatorname{sqrt}}(H z)$} & ضريب نويز هر محور \\
\hline \multicolumn{3}{|c|}{$\cdot, \cdot 1 \%$} & ضريب غير خطى هر محور \\
\hline \multicolumn{3}{|c|}{$\cdot, \cdot 1 \%$} & ضريب هيسترزيس هر محور \\
\hline \multicolumn{3}{|c|}{ - } & ضريب نفوذيذيرى هر محور \\
\hline \multicolumn{3}{|c|}{${ }^{4} n T /{ }^{\circ} \mathrm{C}$} & ضريب دمايى باياس \\
\hline \multicolumn{3}{|c|}{${ }^{9} n T /$ year } & ضريب بلند مدت باياس \\
\hline \multicolumn{3}{|c|}{ r...nnT } & محدوده - محد \\
\hline \multicolumn{3}{|c|}{ 1.. Hz } & بـ بهناى باند \\
\hline \multicolumn{3}{|c|}{19 bit } & تعداد بيت گسسته سازى \\
\hline \multicolumn{3}{|c|}{$\begin{array}{l}D \\
=\left(\begin{array}{lll}0.01 & 0.01 & 0.01 \\
0.01 & 0.02 & 0.01 \\
0.01 & 0.01 & 0.01\end{array}\right.\end{array}$} & D = LS ماتريس \\
\hline & $\Delta \mathrm{mrad}$ & & خطاى نصب داخلى براى هر محور \\
\hline
\end{tabular}
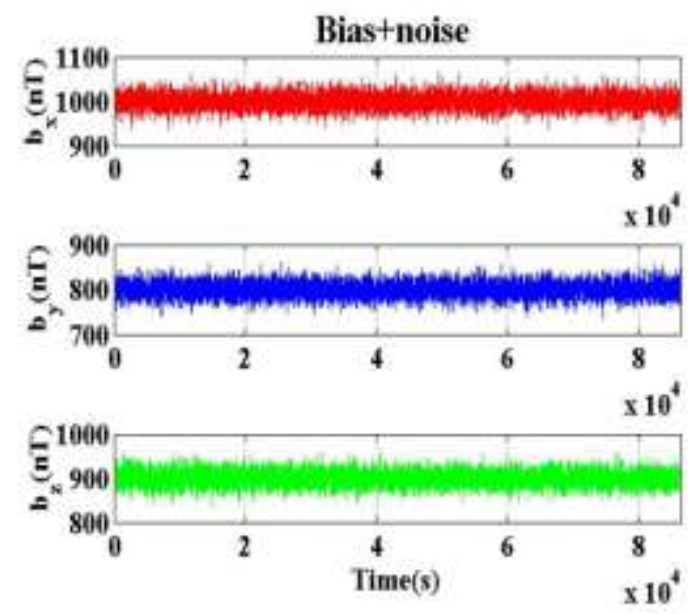

شكل ه: مجموع باياس و نويز حسكر مغناطيسى $\hat{z}_{k+1}(-)=\sum_{i=0}^{18} w_{i}^{(m)} z_{i, k+1}$

همجينين كوواريانس اندازه گيرى و ماتريس همبستخى متقابل به شكل زير

$P_{z z, k+1}=\sum_{i=0}^{18} w_{i}^{(c)}\left(z_{i, k+1}-\hat{z}_{k+1}(-)\right)\left(z_{i, k+1}-\hat{z}_{k+1}(-)\right)^{T}+\sigma_{k+1}$

$P_{x z, k+1}=\sum_{i=0}^{18} w_{i}^{(c)}\left(\chi_{i, k+1}-\hat{x}_{k+1}(-)\right)\left(z_{i, k+1}-\hat{z}_{k+1}(-)\right)^{T}$

در نهايت بهره كالمن فيلتر، حالتهاى بسين و ماتريس كوواريانس خطاى حالت يسين به صورت زير است:

$K_{k+1}=P_{x z, k+1} P_{z z, k+1}^{-1}$

$(9 \mathrm{~V})$

$\hat{x}_{k+1}(+)=\hat{x}_{k+1}(-)+K_{k+1}\left(z_{k+1}-\hat{z}_{k+1}(-)\right)$

$P_{x x, k+1}(+)=P_{x x, k+1}(-)-K_{k+1} P_{z z} K_{k+1}^{T}$

روابط (ه9) تا (99) آنقدر تكرار مىشوند تا الكوريتم به مقدار مطلوب همخرا شود.

9-Y-مرحله دوم از الكوريتم فيلتر كالمن دو مرحلهاى اين مرحله براى استخر اج يارامترهاى خطاى نصب مورد استفاده قرار

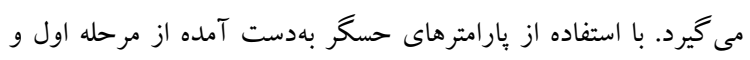

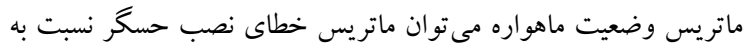

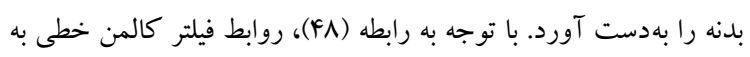

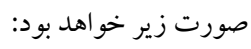

$K_{k}=P_{k} W_{k+1}^{T}\left(W_{k+1} P_{k} W_{k+1}^{T}+(I+D)^{-1} \Sigma_{k+1}\right)^{-1}$

$\hat{x}_{k+1}=\hat{x}_{k}+K_{k}\left(\vec{V}_{k+1}-W_{k+1} \hat{x}_{k}\right)$

$P_{k+1}=\left(I-K_{k} W_{k+1}\right) P_{k}$

روابط (•V) تا (VY) تكرار مىشوند تا الكوريتم به مقدار مطلوب همخرا

شود.

Y- نتايج شبيهسازى

در اين بخش هدف از شبيهسازى بررسى هر يك از روشها از نظر

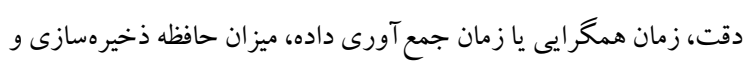

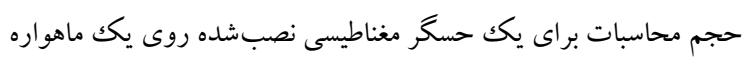

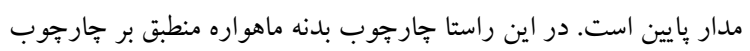

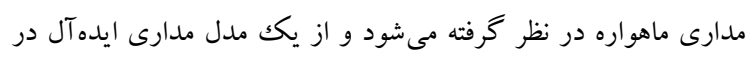

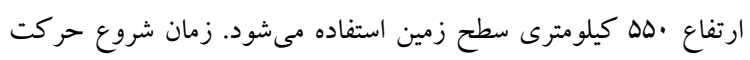

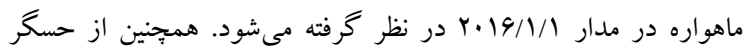

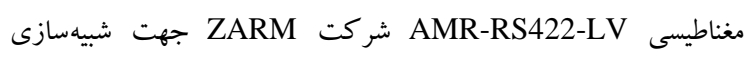




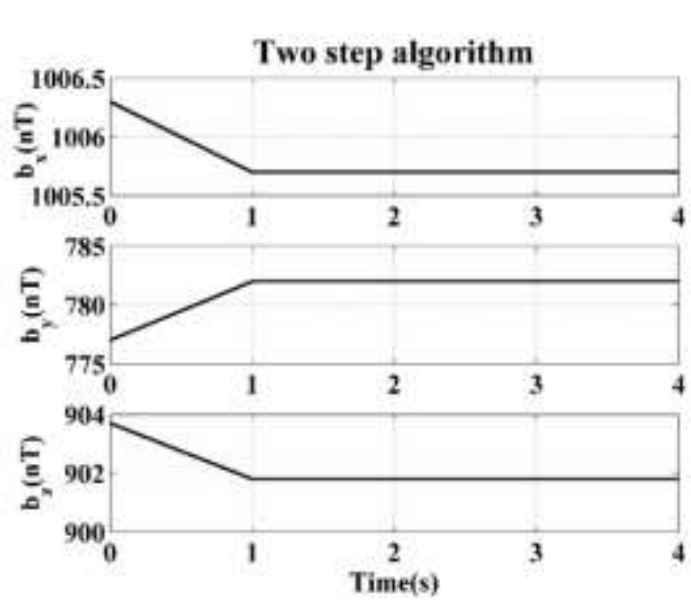

شكل V: نتايج الكوريتم حداقل مربعات غيرخطى براى باياس حسكر مغناطيسى

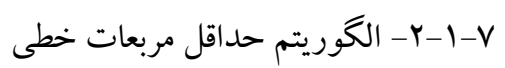

با اجراى الكوريتم حداقل مربعات خطى، ماتريس خطاى نصب به -

دست آمده بهصورت زير است:

$$
R_{b o d y}^{\text {sensor }}=\left(\begin{array}{ccc}
0.9992 & 0.0365 & -0.0190 \\
-0.0358 & 0.9992 & 0.0189 \\
0.0199 & -0.0192 & 0.9996
\end{array}\right)
$$

بر اساس اين ماتريس، زواياى خطاى نصب مطابق با جدول (F) است. طبق

\begin{tabular}{|c|c|c|}
\hline \multicolumn{3}{|c|}{ جدول f: زواياى ماتريس خطاى نصب } \\
\hline مقدار بهدست آمده & مقدارصحيح & 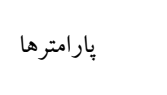 \\
\hline$(1 / \cdot \wedge, r / \cdot q, 1 / \cdot \wedge)$ & $(1, r, 1)$ & $\left(\begin{array}{lll}\theta & \phi & \psi\end{array}\right)^{T}$ \\
\hline
\end{tabular}
جدول، ميانگين خطا برابر هـ•, · درجه است كه دقت قابل قبولى است.

Y-V

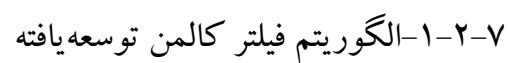
با زمان نمونهبردارى لا ثانيه و ده مرتبه اجراى الكوريتم فيلتر

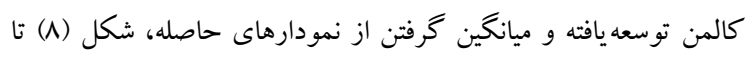

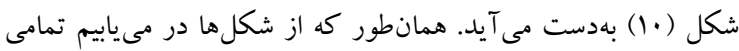

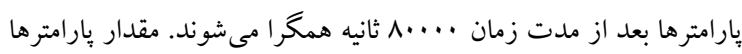

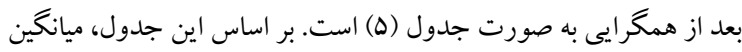

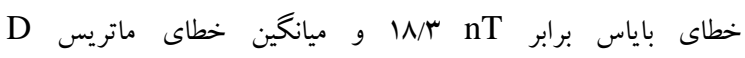

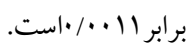

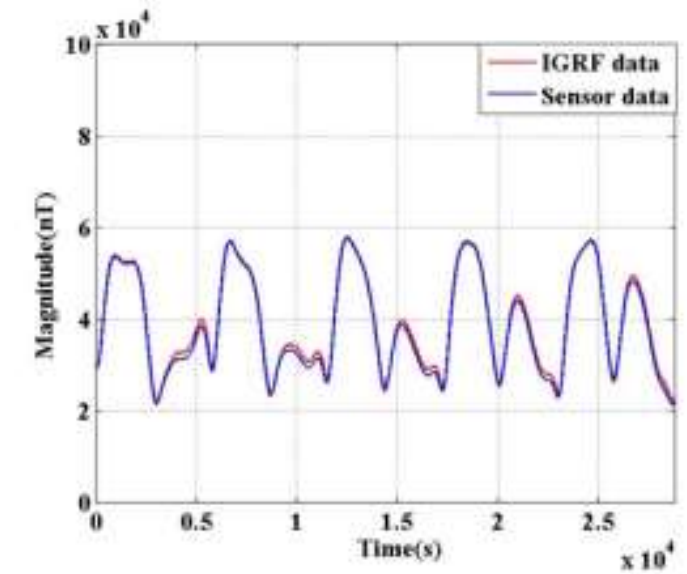

شكل 9: اندازه بردار خروجى حسگر و اندازه بردار مدل ميدان مغناطيسى مرجع

-1-V

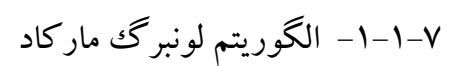

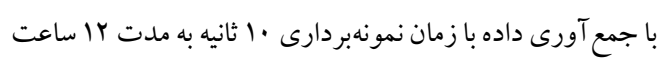

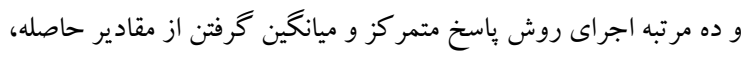

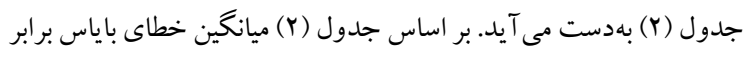
Il nT

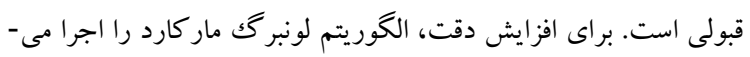

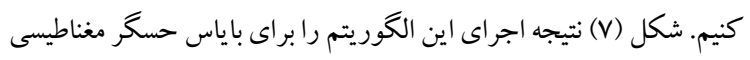

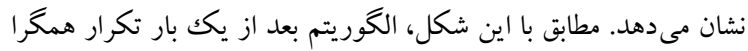

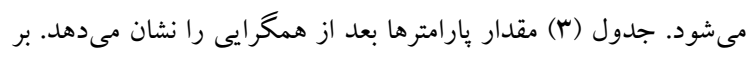

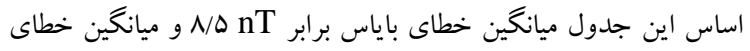
ماتريس D برابر 19 |... • است كه نشان مىدهد دقت نسبت به مرحله اول

\begin{tabular}{|c|c|c|}
\hline مقداربهدست آمده & مقدارصحى & يارامترها \\
\hline$(1 \cdots q / r, V V V, q \cdot r / V)$ & $(1 \cdots \cdot \wedge \cdot \cdot \cdot, \cdot \cdot \cdot)$ & $\left(\begin{array}{lll}b_{1} & b_{2} & b_{3}\end{array}\right)^{T}$ \\
\hline$(\cdot / \cdot 1 \cdot r, \cdot / \cdot 190, \cdot / \cdot 1 \cdot \cdot)$ & $(\cdot / \cdot 1, \cdot / \cdot r, \cdot / \cdot 1)$ & $\left(\begin{array}{lll}D_{11} & D_{22} & D_{33}\end{array}\right)^{T}$ \\
\hline$(\cdot / \cdot 1 \cdot r, \cdot / \cdot 1 \cdot r, \cdot / \cdot \bullet q q)$ & $(\cdot / \cdot 1, \cdot / \cdot 1, \cdot / \cdot 1)$ & $\left(\begin{array}{lll}D_{12} & D_{13} & D_{23}\end{array}\right)^{T}$ \\
\hline
\end{tabular}

$$
\text { بيشتر شده است. }
$$

\begin{tabular}{|c|c|c|}
\hline \multicolumn{3}{|c|}{ جدول س: نتايج الكوريتم هاسخ لونبر گك مار كاد } \\
\hline مقداربهدست آمده & مقدارصحيح & 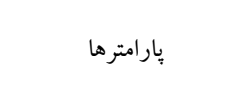 \\
\hline$(1 \cdot \cdot \Delta / V, V \wedge Y / \cdot, q \cdot 1 / \Lambda)$ & $(1 \cdots, \wedge \cdot \cdot, q \cdot \cdot)$ & $\left(\begin{array}{lll}b_{1} & b_{2} & b_{3}\end{array}\right)^{T}$ \\
\hline$(\cdot / \cdot 1 \cdot r, \cdot / \cdot 19 V, \cdot / \cdot 1 \cdot \cdot)$ & $(\cdot / \cdot 1, \cdot / \cdot r, \cdot / \cdot 1)$ & $\left(\begin{array}{lll}D_{11} & D_{22} & D_{33}\end{array}\right)^{T}$ \\
\hline$(\cdot / \cdot 1 \cdot 1, \cdot / \cdot 1 \cdot r, \cdot / \cdot \cdot q \Lambda)$ & $(\cdot / \cdot 1, \cdot / \cdot 1, \cdot / \cdot 1)$ & $\left(\begin{array}{lll}D_{12} & D_{13} & D_{23}\end{array}\right)^{T}$ \\
\hline
\end{tabular}




\begin{tabular}{|c|c|c|}
\hline مقداربهدست آمده & مقدارصحيح & ي بارامترها \\
\hline$(1 \cdot \mid r / v, V 9 q / 9, q \cdot V / \Lambda)$ & $(1 \cdots, \wedge \cdot \cdot, q \cdot \cdot)$ & $\left.\begin{array}{lll}b_{1} & b_{2} & b_{3}\end{array}\right)^{T}$ \\
\hline$(\cdot / \cdot \wedge \wedge, \cdot / \cdot 191, \cdots \cdot 1 \cdot 1)$ & $\left(\cdot / \cdot 1, \cdots / r_{1}, / \cdot 1\right)$ & $\left(\begin{array}{lll}D_{11} & D_{22} & D_{33}\end{array}\right)^{T}$ \\
\hline$(\cdot / \cdot \wedge r, \cdot / \cap \cdot r, \cdots / \cdot q \mathrm{qV})$ & $(\cdot / \cdot 1, \cdot / \cdot 1, \cdot / \cdot 1)$ & $\left(\begin{array}{lll}D_{12} & D_{13} & D_{23}\end{array}\right)^{T}$ \\
\hline
\end{tabular}

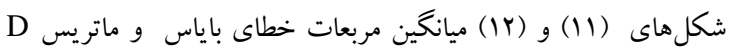

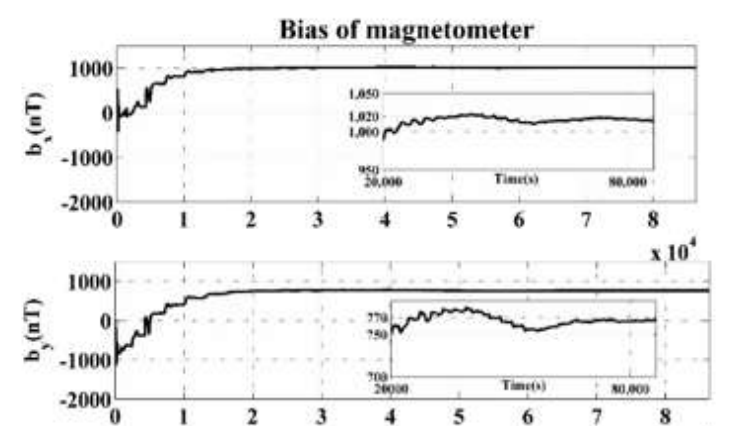

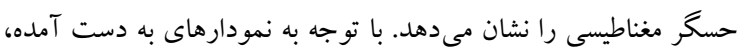

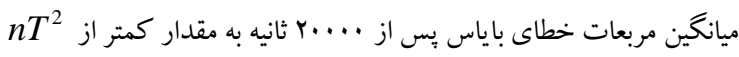

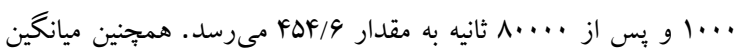

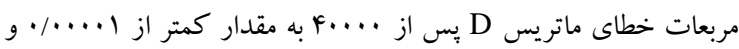

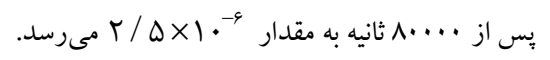

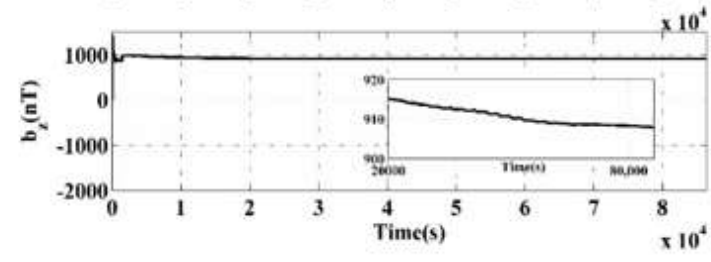
شكل م: تخمين باياس با استفاده از الكوريتم فيلتر كالمن توسعه يافته
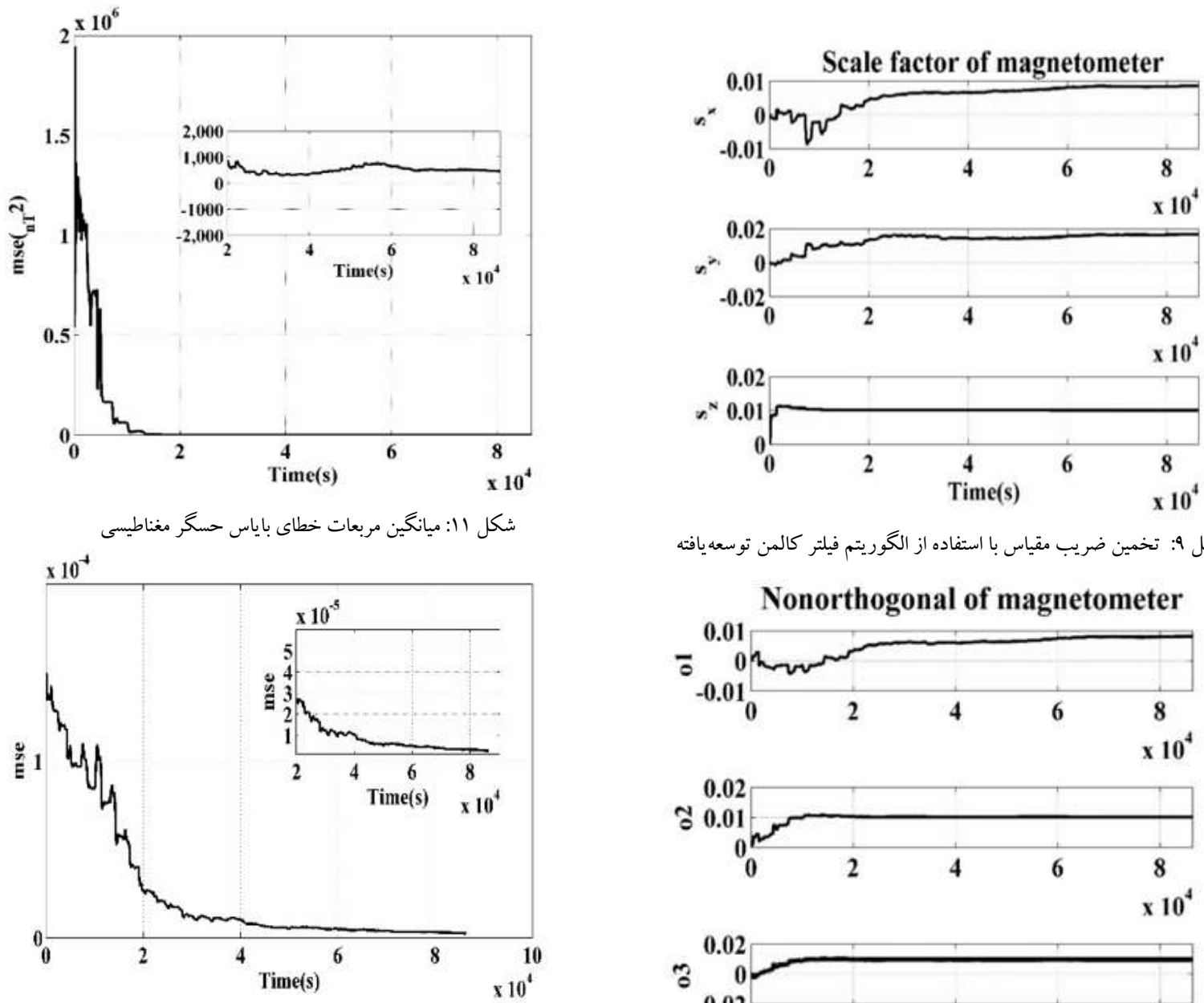

شكل ه: تخمين ضريب مقياس با استفاده از الكوريتم فيلتر كالمن توسعهافته
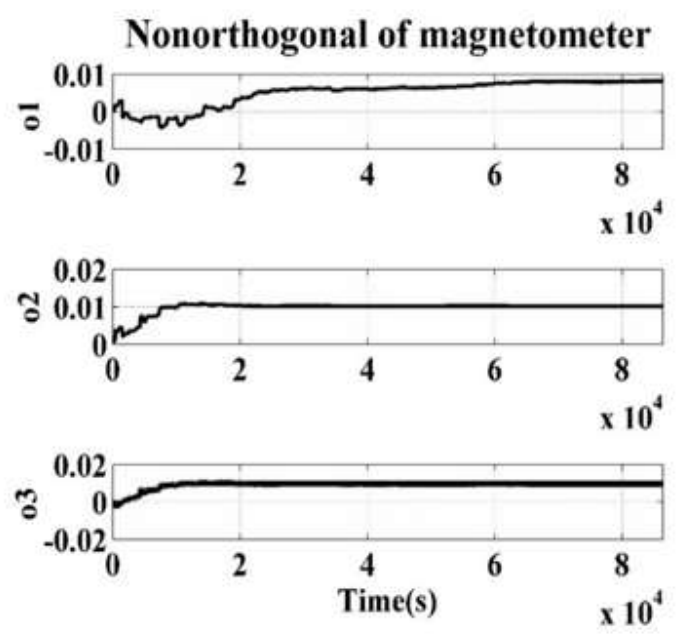

شكل · ا: تخمين عدم تعامد با استفاده از الكوريتم فيلتر كالمن توسعهيافته

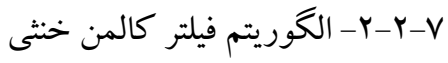



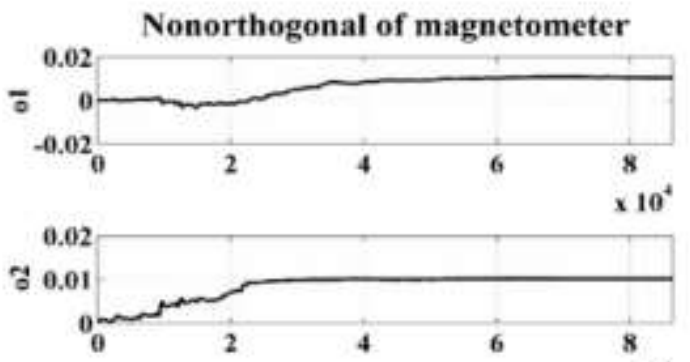

$\times 10^{4}$

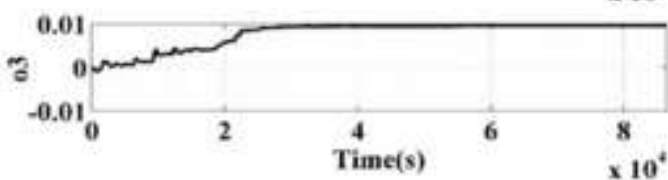

شكل ها: تخمين عدم تعامد با استفاده از الخوريتم فيلتر كالمن خنثى

\begin{tabular}{|c|c|c|}
\hline مقداربهدست آمده & مقدارصحيح & يار امترها \\
\hline$(1 \cdot \cdot q / q, b V V / r, q \cdot q / r)$ & $(1 \cdots, \wedge \cdots, q \cdots)$ & $\left(\begin{array}{lll}b_{1} & b_{2} & b_{3}\end{array}\right)^{T}$ \\
\hline$(\cdot / \cdot 1 \cdot 1, \cdot / \cdot r \cdot \cdot, \cdot / \cdot 1 \cdot \cdot)$ & $(\cdot / \cdot 1, \cdot / \cdot r, \cdot / \cdot 1)$ & $\left(\begin{array}{lll}D_{11} & D_{22} & D_{33}\end{array}\right)^{T}$ \\
\hline$(\cdot / \cdot 1 \cdot k, \cdot / \cdot 1 \cdot 1, \cdot / \cdot \cdot q v)$ & $(\cdot / \cdot 1, \cdot / \cdot 1, \cdot / \cdot 1)$ & $\left(\begin{array}{lll}D_{12} & D_{13} & D_{23}\end{array}\right)^{T}$ \\
\hline
\end{tabular}

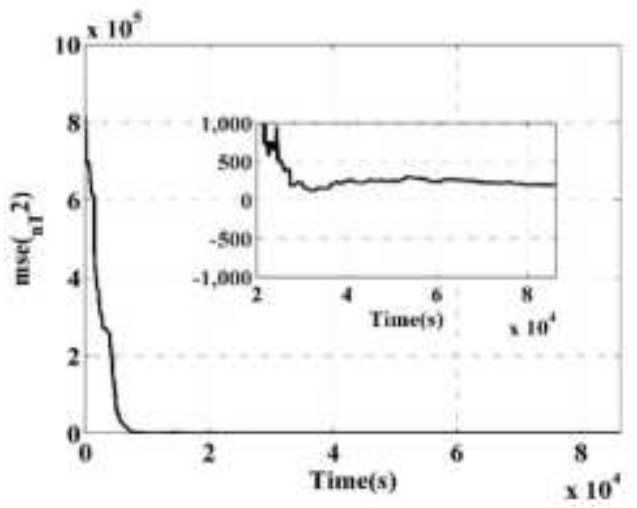

شكل 19: ميانگين مربعات خطاى باياس حسگر مغناطيسى

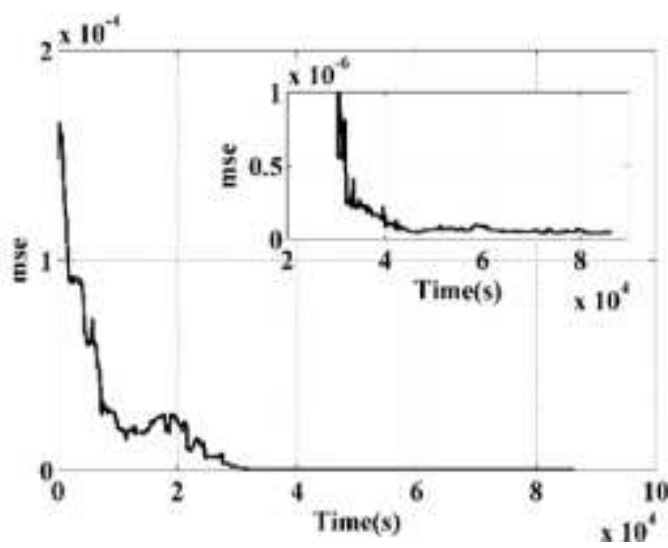

با زمان نمونهبردارى و اجراى ده مرتبه الخوريتم فيلتر كالمن خنثى و

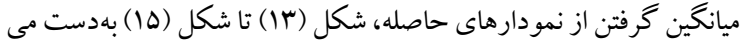
آيد. همانطور كه از شكل ها در مى يابيم تمامى يار امترها بعد از مدت زمان A.... . جدول (9) است. بر اساس اين جدول، ميانگين خطاى باياس برابر nT

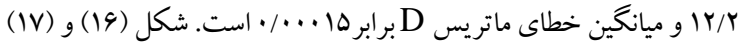
ميانگين مربعات خطاى باياس حسكر مغناطيسى را نشان مىدهد. با توجه به نمودار بهدست آمده ميانگين مربعات خطا يس از ....... ثانيه به مقدار

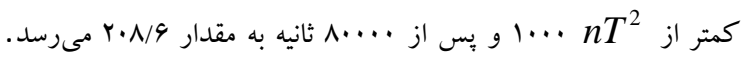

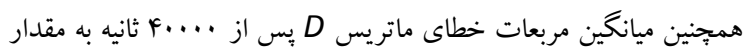

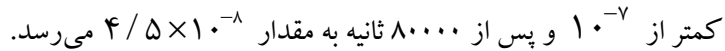
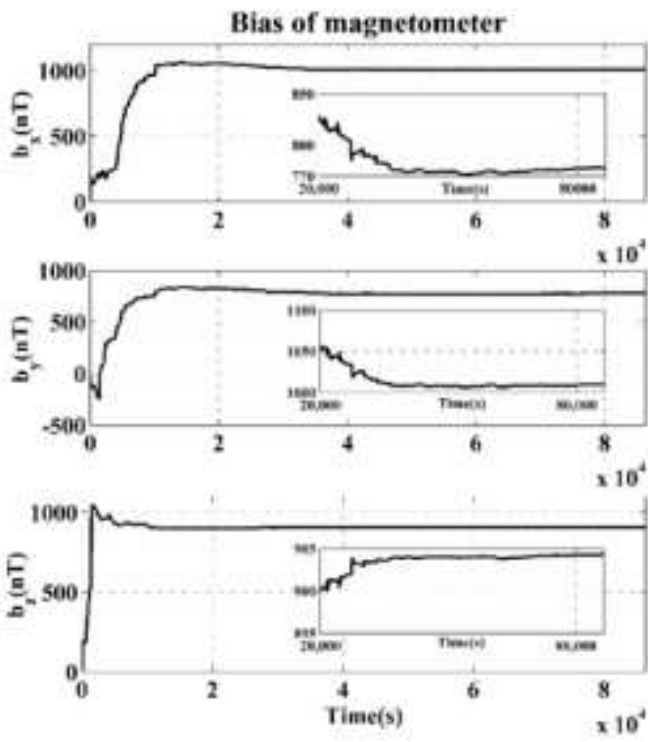
شكل سا: تخمين باياس با استفاده از الكوريتم فيلتر كالمن خنثى
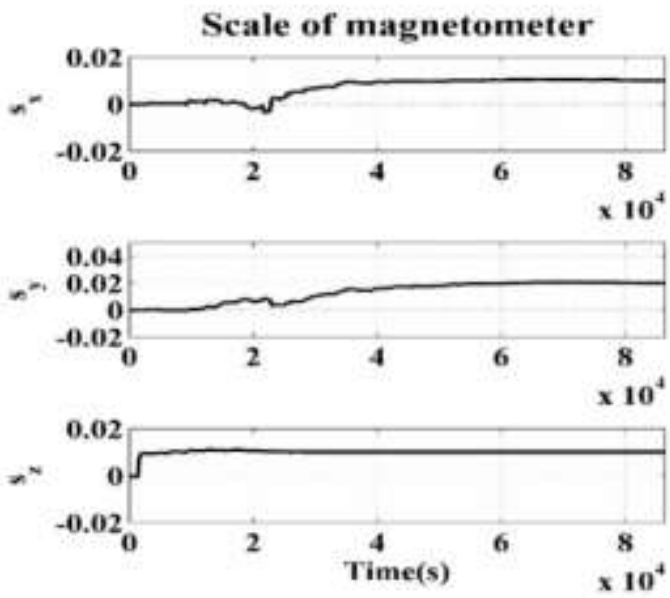

شكل \& ا: تخمين ضريب مقياس با استفاده از الكوريتم فيلتر كالمن خنثى 


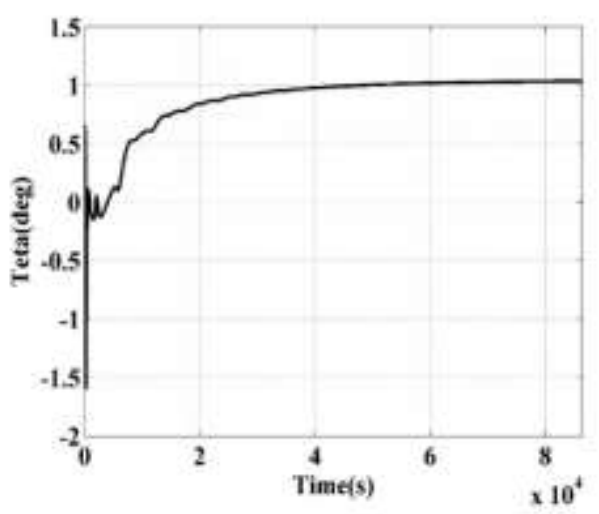

شكل ·r: تخمين زاويه ياو با استفاده از الكوريتم فيلتر كالمن خطى

جدول من نتايج شبيه سازى الخوريتمهاى كاليبراسيون روى برد حسخر مغناطيسى زمان

\begin{tabular}{|c|c|c|c|c|}
\hline محاسبات & حجم & همكر زمايى /زمان & $\begin{array}{c}\text { (بSE) } \\
\text { (باياس) }\end{array}$ & نام روش \\
\hline $\begin{array}{l}1 / \Delta \\
\text { ثانيه }\end{array}$ & 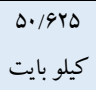 & rا ساعت & $\begin{array}{l}19 F / 1 \\
n T^{2}\end{array}$ & متاسخ \\
\hline $\begin{array}{l}4 . \\
\text { ثانيه }\end{array}$ & 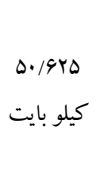 & rا ساعت & $\begin{array}{l}n T^{2} \\
119 / 9\end{array}$ & 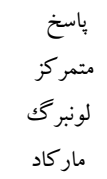 \\
\hline $\begin{array}{l}\text { Vانيه } \\
\text { t/r }\end{array}$ & $\begin{array}{l}\text { بايت } \\
\end{array}$ & TF Mاع TF & $\begin{array}{l}n T^{2} \\
\forall \Delta F / 9\end{array}$ & فيلتر كالمن \\
\hline $\begin{array}{l}\text { ثانيه } \\
\text { | } 19 / 9\end{array}$ & بايت & FF Mاعت & $\begin{array}{l}n n T^{2} \\
r \cdot N / 9\end{array}$ & فيلتر كالمن \\
\hline
\end{tabular}

خلاصه نتايج شبيه سازى روش ها در جدول (1) آورده شده است. همان-

طور كه ديده مى شود از بين روشهاى خارج از خط، روش بـ باسخ متمر كز

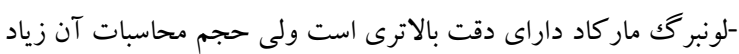

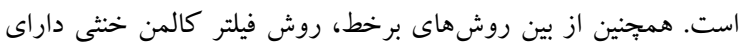

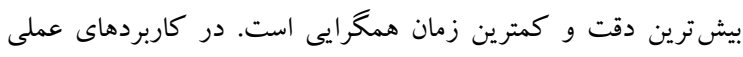
معمولا روش هاى برخط به دليل نياز به حافظه ذخيرهسازى كم و زمان دمان

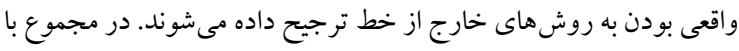

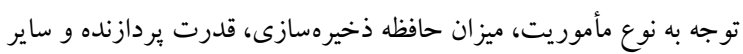

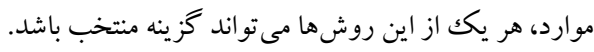

\section{1- نتيجه Fيرى}

در اين مقاله يكك مدل از حسخر مغناطيسى مورد بررسى قرار گرفت كه بر خلاف مدلهاى كذشته شامل اثر غير خطى، اثر هيسترزيس، اثر كوانتيزه

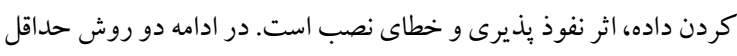

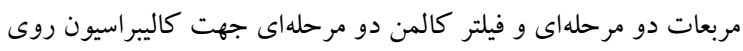

شكل VI: ميانگين مربعات خطاى ماتريس D حسكر مغناطيسى

$$
\text { r-Y-V }
$$

با انتخاب الكَوريتم فيلتر كالمن خنثى و ده مرتبه اجر ایى همزمان آن با فيلتر كالمن خطى جهت بهدست آوردن خطاى نصب و ميانگين كرفتن

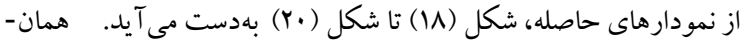

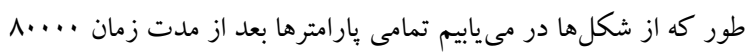

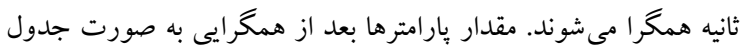

جدولV: زواياى ماتريس خطاى نصب

\begin{tabular}{|c|c|c|}
\hline مقداربهدست آمده & مقدارصحيح & ي پِارامترها \\
\hline$(1 / \cdot r, r / 10,1 / 1 \cdot)$ & $(1, Y, 1)$ & $\left(\begin{array}{lll}\theta & \phi & \psi\end{array}\right)^{T}$ \\
\hline
\end{tabular}

بنابراين ميانگين خطا برابر 9.

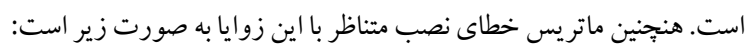
$R_{\text {body }}^{\text {sensor }}=\left(\begin{array}{ccc}0.9992 & 0.0375 & -0.0181 \\ -0.0356 & 0.9991 & 0.0193 \\ 0.0203 & -0.0195 & 0.9997\end{array}\right)$

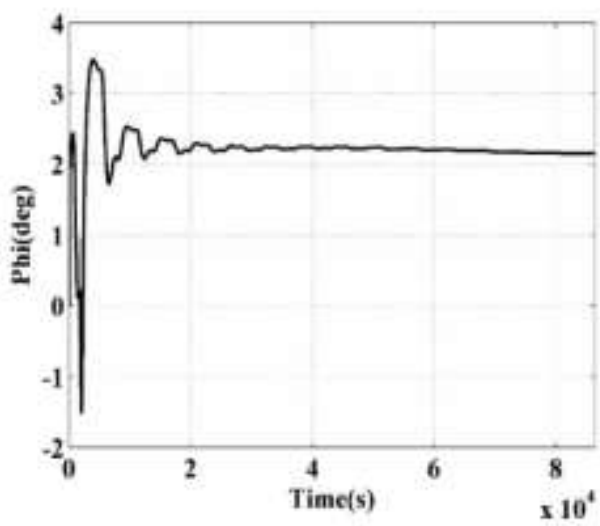
شكل 11: تخمين زاويه رول با استفاده از الكوريتم فيلتر كالمن خطى

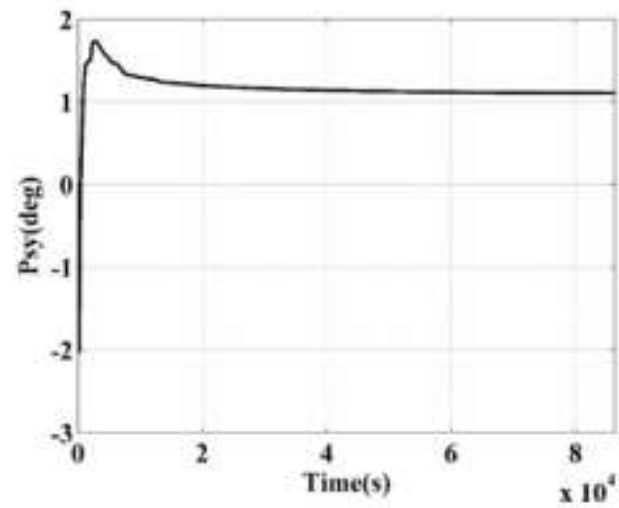

شكل 19: تخمين زاويه بيجج با استفاده از الكوريتم فيلتر كالمن خطى 
[10] Yang, D., You, Z., Li, B., Duan, W., \& Yuan, B., "Complete Tri-Axis Magnetometer Calibration with a Gyro Auxiliary”, Sensors, 17(6), 2017.

[11] Jung H, Psiaki ML, "Tests of magnetometer/sun-sensor orbit determination using flight data", J Guide, Control Dyn, pp. 582-90, 2002.

[12] Takahashi F, Shimizu H, Matsushima M, Shibuya H, Matsuoka S, Nakazawa S, et al, "In-orbit calibration of the lunar magnetometer onboard SELENE (KAGUYA)", Earth Planets Space, pp. 1269-74, 2009.

[13] Li W, Wang JL, "Magnetic sensors for navigation applications: an overview", J Navig, pp.263-75, 2014.

[14] F.L. Markley, J.L. Crassidis, "Fundamental of spacecraft Attitude Determination and Control”, Springer, 2014.

[15] Alonso R, Shuster MD, "Attitude-independent magnetometer-bias determination: a survey", J Astronaut Sci, pp. 453-75, 2002.

[16] Alonso R, Shuster MD, "TWOSTEP: a fast robust algorithm for attitude-independent magnetometer-bias determination”, J Astronaut Sci, pp. 433-51, 2002.

[17] Alonso R, Shuster MD, "Complete linear attitudeIndependent magnetometer calibration", J Astronaut Sci, pp. 477-90,2002.

[18] Crassidis JL, Lai KL, Harman RR, "Real-time attitudeindependent three-axis magnetometer calibration", J Guid Control Dyn pp. 115-20, 2005.

[19] Soken HE, Hajiyev C, "UKF based in-flight calibration of magnetometers and rate gyros for pico satellite attitude determination”, Asian J Control, pp. 707-15, 2012.

[20] Soken HE, Hajiyev C, "UKF-based reconfigurable attitude parameters estimation and magnetometer calibration", IEEE Trans Aerosp Electron Syst, pp. 261427, 2012.

[21] Juang JC, Tsai YF, Tsai CT, "Design and verification of a magnetometer-based orbit determination and sensor calibration algorithm", Aerosp Sci Technol, pp. 47-54, 2012.

[22] Z. Zhen, X. Jianping, J. Jin, “On-orbit real time magnetometer bias determination for micro-satellite without attitude information", Chinese Journal of Aeronautics, pp. 1503-1509, 2015.

[23] F. Landis Markley, John L. Crassidis, "Fundamental of spacecraft attitude determination and control", Springer, 2014.

[24] Abdelrahman, M., Chang, I., \& Park, S. Y.,"Magnetic torque attitude control of a satellite using the statedependent Riccati equation technique", International Journal of Non-Linear Mechanics, 46(5), pp. 758-771, 2011.

[25] E. Thébault., C.C. Finlay, C.D. Beggan, P. Alken, J. Aubert, O. Barrois, F. Bertrand, T. Bondar, A. Boness, L. Brocco, E. Canet, "International geomagnetic reference field: the 12th generation. Earth", Planets and Space, vol. 67, no. 1, pp.1-19, 2015.

$$
\begin{aligned}
& \text { برد ارائه شد. در روش حداقل مربعات دو مرحلهاى در مرحله اول با به - }
\end{aligned}
$$

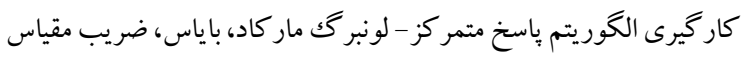

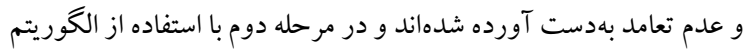

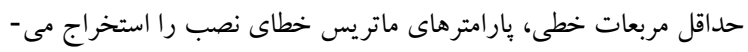

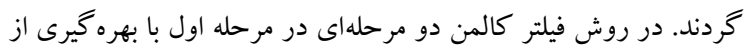

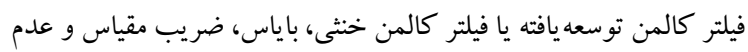

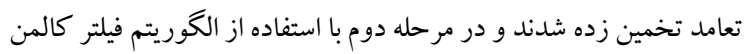

$$
\begin{aligned}
& \text { خطى، بارامترهاى ماتريس خطاى نصب را محاسبه گرديدند. نتايج شبيه- }
\end{aligned}
$$

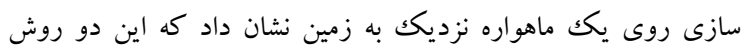

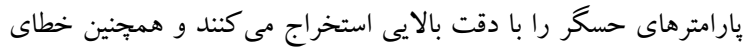

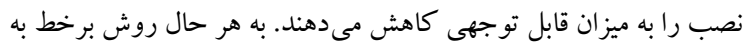

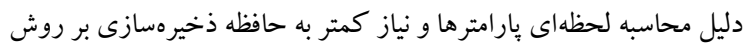

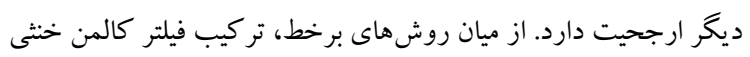

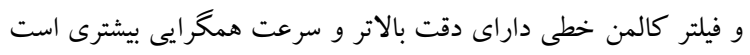

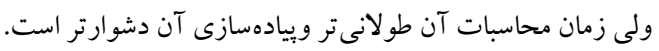

$$
\begin{aligned}
& \text { مر اجع }
\end{aligned}
$$

[1] Jin J, Baoyin HX, Li JF, “Attitude scheme for satellite with defective inertia characteristic", Aircr Eng Aerosp Technol, pp. 422-31, 2013

[2] Ran DC, Sheng T, Cao L, Chen XQ, Zhao Y, "Attitude control system design and on-orbit performance analysis of nano-satellite", Chin J Aeronaut, pp. 593-601, 2013.

[3] Han K, Wang H, Tu BJ, Jin ZH, "Pico-satellite autonomous navigation with magnetometer and sun sensor data", Chin J Aeronaut, pp. 46-54, 2011.

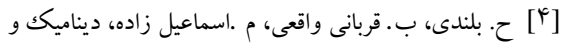

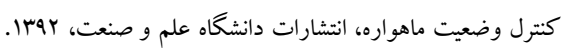

[5] D. Gebre-Egziabher, G. Elkaim, J. Powell, B. Parkinson, "Calibration of strapdown magnetometers in magnetic field domain", J. Aerospace Eng, 19 (2), pp. 87-102, 2006.

[6] T. Pylvanainen, "Automatic and adaptive calibration of 3D field sensors", Applied Mathematical Modelling 32, pp. 575-587, 2007.

[7] H. Pang, D. Chen, M. Pan, S. Luo, Q. Zang, J. Li, C. Wan, J. Wang, F. Luo and W. Wang, "Calibration of three-axis magnetometer with differential evolution algorithm," Journal of Magnetism and Magnetic Materials, vol 346, pp. 5-10, 2013.

[8] H. Pang, D. Chen, M. Pan, S. Luo, Q. Zang, J. Li and F. Luo," A New Calibration Method of Three Axis Magnetometer with Nonlinearity Suppression,'IEEE TRANSACTION ON MAGNET, vol. 49, no. 9, 2013.

[9] S.A.H Tabatabaei, A. Gluhak and R. Tafazoli,"A Fast Calibration Method for Triaxial Magnetometers," IEEE Transaction on Instrument and Measurement, vol. 62, no. 11, pp. 2929-2937, 2013. 